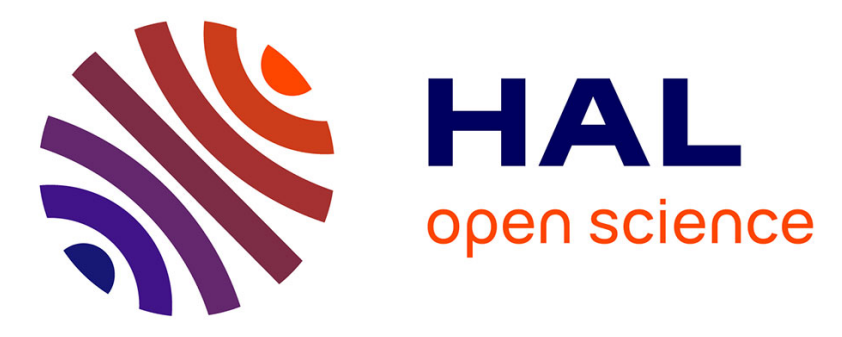

\title{
Static osteogenesis does not precede dynamic osteogenesis in periosteal ossification of Pleurodeles (Caudata, Amphibia) and Pogona (Squamata, Lepidosauria) \\ Jorge Cubo, Mylaine Hui, François Clarac, Alexandra Quilhac
}

\section{To cite this version:}

Jorge Cubo, Mylaine Hui, François Clarac, Alexandra Quilhac. Static osteogenesis does not precede dynamic osteogenesis in periosteal ossification of Pleurodeles (Caudata, Amphibia) and Pogona (Squamata, Lepidosauria). Journal of Morphology, 2017, 278 (5), pp.621-628. 10.1002/jmor.20659 . hal-02298296

\section{HAL Id: hal-02298296 \\ https: / hal.sorbonne-universite.fr/hal-02298296}

Submitted on 26 Sep 2019

HAL is a multi-disciplinary open access archive for the deposit and dissemination of scientific research documents, whether they are published or not. The documents may come from teaching and research institutions in France or abroad, or from public or private research centers.
L'archive ouverte pluridisciplinaire HAL, est destinée au dépôt et à la diffusion de documents scientifiques de niveau recherche, publiés ou non, émanant des établissements d'enseignement et de recherche français ou étrangers, des laboratoires publics ou privés. 
Static osteogenesis does not precede dynamic osteogenesis in periosteal ossification of Pleurodeles (Caudata, Amphibia) and Pogona (Squamata, Lepidosauria)

by

Jorge Cubo $^{1 *}$, Mylaine Hui ${ }^{1}$, François Clarac $^{1,2}$ \& Alexandra Quilhac ${ }^{1}$

${ }^{1}$ Sorbonne Universités UPMC Univ Paris 6, CNRS, Institut des Sciences de la Terre de Paris (ISTeP), 4 place Jussieu - BC 19 - 75005 Paris, France

${ }^{2}$ Département Histoire de la Terre, Museum National D'histoire Naturelle, UMR 7207 (CR2P), Sorbonne Universités, Muséum National d'Histoire Naturelle

(MNHN)/CNRS/UPMC, Bâtiment De Géologie Paris Cedex 05 F-75231, France

*Corresponding author: jorge.cubo_garcia@upmc.fr 
ABSTRACT. Two successive mechanisms have been described in perichondral ossification: (1) in static osteogenesis, mesenchymal cells differentiate into stationary osteoblasts oriented randomly, which differentiate into osteocytes in the same site; (2) in dynamic osteogenesis, mesenchymal cells differentiate into osteoblasts that are all oriented in the same direction and move back as they secrete collagen fibers. This study is aimed at testing the hypothesis that the ontogenetic sequence static then dynamic osteogenesis observed in the chicken and in the rabbit is homologous and was acquired by the last common ancestor of amniotes or at a more inclusive node. For this we analyze the developmental patterns of Pleurodeles (Caudata, Amphibia) and those of the lizard Pogona (Squamata, Lepidosauria). We processed Pleurodeles larvae and Pogona embryos, prepared thin and ultrathin sections of appendicular bones, and analyzed them using respectively light and transmission electron microscopy. We show that static osteogenesis does not precede dynamic osteogenesis in periosteal ossification of Pleurodeles and Pogona. Therefore, the null hypothesis is rejected and according to the parsimony method the ontogenetic sequence observed in the chicken and in the rabbit are convergent. In Pleurodeles and Pogona dynamic osteogenesis occur without a previous rigid mineralized framework, whereas in the chicken and in the rabbit dynamic osteogenesis seems to take place over a mineralized support whether bone (in perichondral ossification) or calcified cartilage (in endochondral ossification). Moreover, in typical dynamic osteogenesis, osteoblasts show an axis (basal nucleus - distal endoplasmic reticulum) perpendicular to the front of secreted unmineralized bone matrix, whereas in Pleurodeles and Pogona this axis is parallel to the bone matrix. 


\section{RESEARCH HIGHLIGHTS}

Contrary to what has been observed in the chicken and in the rabbit, in Pleurodeles and Pogona (1) dynamic osteogenesis occur without a previous rigid mineralized framework, and (2) osteoblasts are polarized with an axis parallel to the bone matrix.

GRAPHICAL ABSTRACT

Please see attached picture.

\section{KEYWORDS}

Bone histology, Parallel fibered bone, Perichondral ossification, Osteocyte, Osteoblast 


\section{INTRODUCTION}

Bone paleohistological analyses are a powerful tool to infer paleobiological features of extinct taxa such as their lifestyle (Canoville and Laurin, 2010; Quémeneur et al., 2013), bone growth rates (Cubo et al., 2012; Legendre et al., 2013) or resting metabolic rates (Legendre et al., 2016). To perform reliable paleobiological inferences, we need a deep knowledge of the mechanisms involved in bone formation. Recent important discoveries show how osteoblasts become osteocytes (Franz-Odendaal et al., 2006) and suggest that two successive processes are involved in perichondral ossification in the tibiae of chick embryos and newborn rabbits (Ferretti et al., 2002; Palumbo et al., 2004; Marotti, 2010). (1) In static osteogenesis (SO), mesenchymal cells differentiate into stationary osteoblasts oriented randomly, which differentiate into osteocytes in the same site (Ferretti et al., 2002; Palumbo et al., 2004; Marotti, 2010). (2) In dynamic osteogenesis (DO), mesenchymal cells differentiate into osteoblasts that are all oriented in the same direction and move back as they secrete collagen fibers, forming a migrating lamina of movable osteoblasts (Ferretti et al., 2002; Palumbo et al., 2004; Marotti, 2010). SO produces woven bone whereas DO produces lamellar or non-lamellar parallel-fibered bone (Stein and Prondvai, 2014; Prondvai et al., 2014).

Considering that SO precedes DO in the perichondral ossification of the chicken and the rabbit, this study is aimed at testing the hypothesis that this ontogenetic sequence is homologous and was acquired by the last common ancestor of amniotes. To do it, we analyzed the developmental patterns of Pleurodeles waltl (Caudata, Amphibia), an outgroup of amniotes, and those of the lizard Pogona vitticeps (Squamata, Lepidosauria), an ectothermic amniote. Results obtained are analyzed in the context of tetrapod phylogeny using parsimony. 


\section{MATERIAL AND METHODS}

We processed 8 Pleurodeles larvae and 17 Pogona embryos (coming from a single lay) obtained through captive breeding. Pleurodeles larvae were euthanized at stages 48 - 55 defined by Gallien and Durocher (1957) and Pogona embryos at stages $12-13$ as defined in the developmental staging series for Anolis by Sanger et al. (2008). Appendicular bones were fixed for $2 \mathrm{~h}$ in a mixture containing $2.5 \%$ glutaraldehyde / $2 \%$ paraformaldehyde in $0.1 \mathrm{M}$ cacodylate buffer. The samples were demineralized by the addition of $5 \%$ EDTA to the fixative for two weeks. The demineralized samples were postfixed with $1 \%$ osmium tetroxide in the cacodylate buffer, dehydrated, and subsequently embedded in Epon. Semi-thin $(1 \mu \mathrm{m})$ sections were stained with toluidine blue $(\mathrm{pH} 4)$, examined using a light microscope Zeiss Axiovert 35, and digitalized through an Olympus camera. Thin $(0.05 \mu \mathrm{m})$ sections were double-stained with uranyl acetate and lead citrate. The grids were viewed in a Zeiss Leo transmission electron microscope with an operating voltage of $80 \mathrm{kV}$. We followed the nomenclature proposed by Franz-Odendaal et al. (2006) who reviewed how osteoblasts become osteocytes, and described eight steps: (1) preosteoblast, (2) preosteoblastic osteoblast, (3) osteoblast, (4) osteoblastic osteocyte (Type I preosteocyte), (5) osteoid-osteocyte (Type II preosteocyte), (6) Type III preosteocyte, (7) young osteocyte and (8) old osteocyte.

\section{RESULTS}

\section{Structural analysis}


We present our histological observations on the perichondral ossification in Pleurodeles and Pogona from the less advanced developmental states to the more advanced ones. In the first state observed in our sample of Pleurodeles larvae, mesenchymal cells form condensations and are on their way of differentiation towards chondrocytes (Fig. 1A). In the next state, we observe in each condensation a population of fully differentiated chondrocytes (Fig. 1B). Elongated, flattened, mesenchymal cells form a perichondrium around these condensations (Fig. 1B). The cartilaginous template grows by both mitoses of chondroblasts (Fig. 1C) and by differentiation of mesenchymal cells into chondrocytes (Fig. 1A). In a more advanced developmental state, spindle shaped mesenchymal cells form a perichondrium at some places around the template, whereas they differentiate into osteoblasts at other places (Fig. 1D). These osteoblasts start producing unmineralized osseous matrix (Fig. 1D) and are organized in a migrating lamina that moves back as osteoblasts secrete collagen fibers (Fig. 1E). Spindle shaped mesenchymal cells lining the lamina of osteoblasts form a thin periosteum at this state (Fig. 1E). Even during the formation of outgrowths (crests or trochanters), osteoblasts form a lamina of migrating cells (Fig. 1F). No evidence for static ossification has been observed in Pleurodeles. In figure 1F we can see evidence for degenerating (necrotic) chondrocytes.

In Pogona embryos, prior to the beginning of ossification, the mesenchymal cells around the cartilaginous template are elongated, flattened and arranged in several concentric layers forming the perichondrium (Fig. 2A). In a more advanced state, a few of these mesenchymal cells differentiate into elongated osteoblasts that lay down a layer of unmineralized bone matrix (osteoid, Fig. 2B). Later on, more rounded osteoblasts characterized by a low nucleus to cytoplasm ratio suggesting 
high secretory activity move back as they secrete collagen fibers, forming a migrating lamina of movable cells (arrows in Fig. 2C, D). A few cells stop producing bone matrix and are "buried" by the secretory activity of neighboring cells but conserve the low nucleus to cytoplasm ratio (Fig. 2C, D). Chondrocytes begin to degenerate; their nuclei are shrunk and the cytoplasm is reduced. Some osteoblasts are included in the thin layer of newly deposited bone (Fig. 2D). Afterwards, as the process goes on, cells buried and located at the inner layers of bone, as well as the lacunae into which they are placed, become smaller and acquire a more flattened shape (Fig 2E). These cells show a higher nucleus to cytoplasm ratio, suggesting that they stopped secreting bone matrix, and became osteocytes after mineralization of the osseous matrix (Fig. 2E). The degeneration process of chondrocytes is more and more noticeable leaving large empty chondrocyte lacunae (Fig. 2E). In Fig. 2F we can observe the first signs of chondroclastic activity. Once the cartilage has been resorbed by the activity of plurinucleated chondroclasts, the medullary cavity (containing abundant blood capillaries) expands through osteoclastic activity. Numerous osteocytes are now included in a thick layer of bone (Fig. 2G, H). Finally, some osteoblasts show a more cuboidal shape and are disposed in a single regular layer at the periphery of the bone matrix (Fig. $2 \mathrm{H}$ ).

\section{Ultrastructural analysis}

The ultrastructural analysis adds precision to the description of the cytological features. We followed the nomenclature proposed by Franz-Odendaal et al. (2006). In the steps before the beginning of the ossification, elongated, flattened mesenchymal cells with a reduced cytoplasm and a flat nucleus, are arranged in concentric layers around the cartilaginous template forming the perichondrium (Fig. 
3A, Pleurodeles). In a more advanced developmental state, the cells closest to the cartilage begin to differentiate into more or less rectangular osteoblasts in which we can see moderately developed rough endoplasmic reticulum. Then, we can observe the first osteoblasts with a well developed rough endoplasmic reticulum (Fig. 3B, Pleurodeles; Fig 3C, Pogona), and numerous mitochondria showing an intense secretory activity (Fig. 3E, Pogona). At this state, a layer of flattened mesenchymal cells form the periosteum (Fig 3C, Pogona). The first filopodia begin to protrude in the bone matrix and connect neighbouring cells (Fig. 3D, Pogona). Preosteoblasts and osteoblasts are all polarized in the same direction: the nucleus and the endoplasmic reticulum are aligned in an axis parallel to the front of the secreted unmineralized bone matrix (Fig. 3B, Pleurodeles and 3C,D, Pogona). The secreted bone extracellular matrix is constituted of typical type I collagen fibers oriented in the same direction (Fig. 3F, Pogona). Here and there some osteoblasts are "buried" by the secretory activity of neighboring cells. Once they are entirely embedded in the bone matrix, Type III preosteocytes show a high nucleus to cytoplasm ratio (Fig. 3G, Pogona). These Type III preosteocytes become osteocytes after mineralization of the osseous matrix. They are connected among them and with the osteoblasts of the periphery by dendrites protruding in a little developed network of canaliculi (Fig. 3D, G, Pogona). When the cartilage has been entirely resorbed, fully differentiated osteoblasts show a typical cuboidal shape (Fig. 3H, Pogona) maintaining an active secretory activity and polarized as previously described: the nucleus and the endoplasmic reticulum are aligned in an axis parallel to the front of the secreted unmineralized bone matrix.

\section{DISCUSSION}


Ferretti et al. (2002), Palumbo et al. (2004) and Marotti (2010) proposed the hypothesis that SO precedes DO in the perichondral ossification in chick embryos and newborn rabbits. Here we test whether this ontogenetic sequence is primitive for amniotes. To do it, we analyzed the first phases of perichondral ossification of Pleurodeles (Caudata, Amphibia), an outgroup of amniotes, and Pogona (Squamata, Lepidosauria), an ectothermic amniote. According to the null hypothesis, we expect to find stationary osteoblasts oriented randomly in the first state of the ossification process. The structural and ultrasructural studies show that, contrary to this prediction, all osteoblasts are polarized in roughly the same direction (Figs. 1 -3). These results refute the null hypothesis. So we conclude that SO does not precede DO in periosteal ossification of Pleurodeles or Pogona. Therefore, in the context of the commonly accepted tetrapod phylogeny (Amphibia (Mammalia (Lepidosauria (Aves)))), and using the parsimony method, our results suggest that (a) the ontogenetic sequence observed in the chicken and in the rabbit according to which SO precedes DO is convergent and (b) the ontogenetic pattern observed in Pleurodeles larvae and Pogona embryos according to which SO does not precede DO corresponds to the primitive condition for both Tetrapoda and Amniota.

At first glance, our observations that all osteoblasts are polarized in roughly the same direction at the beginning of perichondral ossification of Pleurodeles and Pogona correspond to typical DO. However, a careful observation of the ultrastructural pictures shows that the osteoblast polarity described in typical DO (as described in chick embryos and newborn rabbits) differ from the osteoblast polarity observed in Pleurodeles and Pogona. In typical DO the osteoblast polarity involves an axis (basal nucleus - distal endoplasmic reticulum) perpendicular to the front of 
secreted unmineralized bone matrix (Ferretti et al., 2002; Palumbo et al., 2004; Marotti, 2010). In contrast, in Pleurodeles and Pogona the nucleus and the endoplasmic reticulum are aligned but in an axis parallel to the front of the secreted unmineralized bone matrix (Fig. 2). We conclude that perichondral ossification in Pleurodeles and Pogona proceeds through a particular mode of DO (different from that described in the chicken and in the rabbit).

It has been classically assumed that SO produces woven bone whereas DO produces lamellar or non-lamellar parallel-fibered bone (Stein and Prondvai, 2014; Prondvai et al., 2014). Our results are congruent with these observations: here we show that the DO observed in our animal models produces parallel collagen fibers (Fig. 2D).

Ricqlès (1979) described in the innermost layer of the mineralized cortex (near the chondrosseous junction) of a number of fossil tetrapods (e.g., Plourdosteus) a cell phenotype intermediate between typical chondrocytes and typical osteocytes ( $p$. 11 and Fig. 3 of Ricqlès, 1979). We compared the size and shape of osteocyte lacunae described by Ricqlès (1979) with those observed in Pleurodeles and Pogona but we were unable to find this cell phenotype in our sample. As described above, osteoblasts located at the innermost layers of bone become smaller, acquire a more flattened shape, and show a higher nucleus to cytoplasm ratio (suggesting that they stopped secreting bone matrix) (Fig 1E). Future studies will elucidate whether collagen type I and II are intermingled the innermost layer (near the chondrosseous junction) using immunohistochemistry.

Roach (1992) reported evidence for the trans-differentiation of hypertrophic chondrocytes into osteoblasts in the hyperthrophic cartilage zone of 14-days-old chick embryos: positive immunocytochemical staining for type I collagen, osteocalcin, 
osteonectin, osteopontin, as well as alkaline phosphatase activity. Transdifferentiation of hypertrophic chondrocytes into osteoblasts has also been reported in endochondral bone during postnatal growth and in endochondral bone repair in mice (Bahney et al., 2014; Zhou et al., 2014), including mandibular endochondral fracture healing (Wong et al., 2016). Marcucio et al. (2014) concluded that transdifferentiation of hypertrophic chondrocytes into osteoblasts involves a transient pluripotent (stem cell-like) state. Cytologically we were unable to find such transformations in perichondral (periostic) ossification of Pleurodeles or Pogona.

Regarding the terminology, the literature on which this study is based on (Ferretti et al., 2002, 2006; Marotti, 2010; Palumbo et al., 2004) used the expression "perichondral intramembranous ossification". However, we followed Franz-Odendaal et al. (2006), who listed three ossification mechanisms (intramembranous, perichondral and endochondral), and we used the expression "perichondral ossification" (see for instance Huysseune and Sire, 1992, for other occurrences of this mechanism). Ricqlès (1979) raised another terminological issue. According to this author (Ricqlès, 1979) cells lining the cartilaginous template form the perichondrium (Figs. 1B, 2A) and become, by definition, a periosteum once they begin to produce bone matrix (Figs. 1E, 2B). Therefore the ossification mechanism analyzed in this study corresponds topologically to a perichondral ossification (because it occurs around a cartilaginous template) producing perichondral bone. However, histologically this mechanism corresponds to a periosteal ossification (because bone matrix and osteoblasts are produced by the periosteum). Consistently, these terms (perichondral versus periosteal) are used interchangeably.

We are aware that the architecture of the bone being formed differs between on the one hand growing small amphibians and squamates and, on the other hand, 
growing mammals and birds. Cubo et al. (2008) showed (in their figure 2) that growing birds (Anas platyrhynchos) and growing mammals (Cavia porcellus) show bone outgrowths that are lacking in growing small squamates (Podarcis muralis). According to Ferretti et al. (2002, 2006), Marotti (2010) and Palumbo et al. (2004), these outgrowths are formed through static ossification, and end up forming cavities that are filled centripetally through dynamic ossification. The point here is to test the hypothesis suggested by Palumbo et al. (2004) according to which "without preexisting osteocytes only woven bone can form [through SO], because an orderly recruitment of osteocytes can only take place by signals issued by a preexisting osteocyte syncytium ». This statement is not universal because, on the one hand, Ferretti et al. (2006) showed that in endochondral ossification of the tibia and humeri of newborn and young rabbits, SO does not precede DO. These authors concluded that calcified cartilage offer the rigid framework necessary for DO (Ferretti et al., 2006). And on the other hand, we have two arguments suggesting that static osteogenesis never seems to take place in the first steps of the ossification of Pleurodeles or Pogona. First, when outgrowths are present, they are formed by dynamic osteogenesis. For instance, in figure $1 \mathrm{~F}$, a zeugopodial bone of a posterior limb of Pleurodeles shows an outgrowth undoubtedly formed by a migrating lamina of osteoblasts that move back as they secrete collagen fibers. Second, Ferretti et al. (2006) noted that "We wish to stress, however, that one must not confuse static osteogenesis with stationary osteoblasts. (...) At the onset of bone formation, it is necessary for some stationary osteoblasts to be present, independently of whether static or dynamic osteogenesis is taking place, (...) Without these stationary osteoblasts, the first layers of bone tissue would not contain osteocytes". The point here is that, contrary to what occurs in SO, where stationary osteoblasts oriented in 
different directions become osteocytes by a mechanism of self-burial, in DO osteoblasts selected to differentiate into osteocytes are buried by the secretory activity of a movable lamina of osteoblasts all oriented in the same direction. We conclude that in Pleurodeles and Pogona DO occurs even without a rigid mineralized framework, whereas in chick embryos and newborn rabbits it seems to take place over a mineralized support whether bone (as observed in perichondral ossification; Ferretti et al., 2002; Palumbo et al., 2004) or calcified cartilage (as observed in endochondral ossification; Ferretti et al., 2006).

\section{AUTHOR CONTRIBUTIONS}

$\mathrm{JC}$ designed research and wrote the draft of the Ms. AQ performed the ultrastructural study using transmission electron microscopy. JC, MH, FC, AQ performed the structural study using light microscopy and contributed to the final version of the Ms. FC obtained the Pogona eggs and he incubated them until the embryologic stage of interest.

\section{ACKNOWLEDGEMENTS}

We thank Hayat Lamrous and Marie-Claire Lajarille for the preparation of, respectively, the thin and ultrathin sections and to Silvia Gardin for giving us access to microscope facilities. We also thank Pauline Wosinski for her collaboration during her undergraduate training at the Pierre \& Marie Curie University. Ultrathin sections were observed in IBPS IFR 3631 in UPMC, Paris. Finally, we would like to address 
our respectful acknowledgments to "La Ferme Tropicale" 54 rue Jenner 75013 Paris, for providing the Pogona eggs, and to the laboratory of Jean Yves Sire (University Pierre \& Marie Curie, Paris, France) for providing the Pleurodeles larvae, without which this investigation would not have been possible.

\section{LITERATURE CITED}

Bahney C, Hu D, Ferro F, Taylor A, Miclau T, Marcucio R. 2014. Transdifferentiation of hypertrophic chondrocytes during endochondral bone repair by activation of pluripotent stem cell programs. Faseb J 28 (1) Supplement 216.1

Canoville A, Laurin M. 2010. Evolution of humeral microanatomy and lifestyle in amniotes, and some comments on palaeobiological inferences. Biol J Linnean Soc 100:384-406.

Cubo J, Legendre P, de Ricqles A, Montes L, de Margerie E, Castanet J, Desdevises Y. 2008. Phylogenetic, functional, and structural components of variation in bone growth rate of amniotes. Evol Dev 10:217-227.

Cubo J, Le Roy N, Martinez-Maza C, Montes L. 2012. Paleohistological estimation of bone growth rate in extinct archosaurs. Paleobiology 38:335-349.

Ferretti A, Palumbo C, Contri M, Marotti G. 2002. Static and dynamic osteogenesis: two different types of bone formation. Anat Embryol 206:21-29.

Ferretti M, Palumbo C, Bertoni L, Cavani F, Marotti G. 2006. Does static precede dynamic osteogenesis in endochondral ossification as occurs in intramembranous ossification? Anat Rec Part A 288A:1158-1162.

Franz-Odendaal TA, Hall BK, Witten PE. 2006. Buried alive: How osteoblasts become osteocytes. Dev Dyn 235:176-190. 
Gallien L, Durocher M. 1957. Table chronologique du développement chez Pleurodeles waltlii Michah. Bull Biol Fr Belg 91: 97-114.

Huysseune A, Sire J. 1992. Development of Cartilage and Bone Tissues of the Anterior Part of the Mandible in Cichlid Fish - a Light and Tem Study. Anat $\operatorname{Rec} 233: 357-375$.

Legendre LJ, Segalen L, Cubo J. 2013. Evidence for High Bone Growth Rate in Euparkeria Obtained Using a New Paleohistological Inference Model for the Humerus. J Vertebr Paleontol 33:1343-1350.

Legendre LJ, Guénard G, Botha-Brink J, Cubo J. 2016: Paleohistological evidence for ancestral high metabolic rate in archosaurs. Syst Biol, in press.

Marcucio R, Hu D, Yang F, Bahney C, Miclau T, 2014. Transdifferentiation of chondrocytes to osteoblasts during bone fracture healing. Faseb J 30 (1) Supplement 223.1

Marotti G. 2010. Static and dynamic osteogenesis. Italian journal of anatomy and embryology = Archivio italiano di anatomia ed embriologia 115:123-126.

Palumbo C, Ferretti M, Marotti G. 2004. Osteocyte dendrogenesis in static and dynamic bone formation: An ultrastructural study. Anat Rec Part A 278A:474480.

Prondvai E, Stein KHW, de Ricqles A, Cubo J. 2014. Development-based revision of bone tissue classification: the importance of semantics for science. Biol J Linnean Soc 112:799-816.

Quémeneur S, De Buffrenil V, Laurin M. 2013. Microanatomy of the amniote femur and inference of lifestyle in limbed vertebrates. Biol J Linnean Soc 109:644655.

Ricqlès A. de. 1979. Quelques remarques sur l'histoire évolutive des tissus 
squelettiques chez les vertébrés et plus particulièrement chez les tétrapodes. Ann Biol 18 :1-35.

Roach H. 1992. Transdifferentiation of Hypertrophic Chondrocytes into Cells Capable of Producing a Mineralized Bone-Matrix. Bone Miner 19:1-20.

Sanger TJ, Losos JB, Gibson-Brown JJ. 2008. A developmental staging series for the lizard genus Anolis: A new system for the integration of evolution, development, and ecology. J Morphol 269:129-137.

Stein K, Prondvai E. 2014. Rethinking the nature of fibrolamellar bone: an integrative biological revision of sauropod plexiform bone formation. Biol Rev 89:24-47. Wong SA, Hu D, Miclau T, Bahney C, Marcucio R. 2016. Trans differentiation of chondrocytes to osteoblasts during endochondral ossification in the healing mandible. Faseb J 30 (1) Supplement 1039.11

Zhou X, von der Mark K, Henry S, Norton W, Adams H, de Crombrugghe B. 2014. Chondrocytes Transdifferentiate into Osteoblasts in Endochondral Bone during Development, Postnatal Growth and Fracture Healing in Mice. PLoS Genet 10:e1004820.

\section{FIGURE LEGENDS}

Fig. 1. Thin cross-sections of appendicular skeletal elements of stage 55 Pleurodeles larvae. A. Skeletal element of the posterior autopod. Mesenchymal cells form condensations and differentiate into chondrocytes (big asterisk). Mesenchymal cells around these condensations are either spindle shaped and form the perichondrium (arrow) or globular and differentiate into chondrocytes (arrow-head). B. Skeletal element of the posterior autopod. Fully differentiated chondrocytes (arrow-head) form 
a cartilaginous template. Flattened mesenchymal cells (arrow) are lining this template forming the perichondrium. C. Skeletal element of the posterior autopod. Evidence for chondroblast mitosis (arrow-heads). The perichondrim is formed by several layers of flattened mesenchymal cells (arrow). D. Anterior zeugopodial bone. Two cellular types are observed around the cartilaginous template: spindle shaped mesenchymal cells forming the perichondrium (arrow) at some places, and osteoblasts (arrowhead) secreting unmineralized osseous matrix (small asterisk) at other places. E. Humerus. Osteoblasts (arrow-heads) produce unmineralized osseous matrix (small asterisk) and move back forming a migrating lamina. Spindle shaped mesenchymal cells form a thin periosteum (arrows). F. Posterior zeugopodial bone. When osteoblasts stop secreting bone matrix (arrow-heads), they are buried by the secretory activity of neighboring osteoblasts (arrow). Outgrowth (big asterisk) formed by migrating osteoblasts. We can observe evidence for degenerative (necrotic) chondrocytes. Scale bar equals $50 \mu \mathrm{m}$ for all pictures

Fig. 2. Thin cross-sections of appendicular skeletal elements of stages 12-13 Pogona embryos. A. Skeletal element of the posterior autopod. The cartilaginous template (asterisk) is surrounded by concentric layers of elongated, flattened, mesenchymal cells forming the perichondrium (arrow). B. Skeletal element of the anterior autopod. Elongated osteoblasts (arrow) lay down a layer of unmineralized bone matrix (osteoid, small asterisk). C. Skeletal element of the posterior autopod. Osteoblasts (arrows) move back as they secrete collagen fibers. Here and there, some of them stop producing bone matrix and are "buried" by the secretory activity of neighboring cells (arrowheads). D. Skeletal element of the posterior autopod. Ossification as in C with some osteoblasts showing a high secretory activity (arrows) and others that 
have been buried by the secretory activity of neighboring osteoblasts (arrow-heads). Here chondrocytes begin to degenerate (they show a necrotic aspect). E. Skeletal element of the posterior autopod. The process of chondrocyte degeneration continues. Osteocytes located at the inner layer of bone become smaller and acquire a more flattened shape (arrows). F. Skeletal element of the posterior autopod. First signs of chondroclastic activity (asterisk). G. Humerus. Once the entire cartilage has been resorbed, the expansion of the medullary cavity occurs by osteoclastic activity (arrow and close-up). The arrowhead shows rests of cartilage. H. Anterior zeugopodial bone. We can observe a layer of fully differentiated cuboidal osteoblasts (arrow head), a plurinucleated chondroclast (arrow) and a blood capillary (asterisk). Scale bar equals $50 \mu \mathrm{m}$ for all pictures but for the close-up,.

Fig. 3. Ultra-thin cross-sections of appendicular skeletal elements of Pleurodeles larvae (A, B) and Pogona embryos (C-H). Nomenclature for the transition from osteoblasts to osteocytes is taken from Franz-Odendaal et al. (2006). A. Spindle shaped mesenchymal cell forming the perichondrium (arrow-head). Cartilage matrix (Ca) in the bottom right corner. B. Osteoblast (arrow) secreting unmineralized bone matrix (osteoid; asterisk). Pre-osteoblast (arrow-head). C. An elongated, flattened, mesenchymal cell (arrow-head) forming the periosteum, a pre-osteoblast (small arrow) and an osteoblast (big arrow) secreting bone matrix (asterisk). D. Concentric layers of pre-osteoblasts (small arrows). We can observe a osteoblast (upper big arrow) and an osteoblastic osteocyte (Type I preosteocyte; lower big arrow) showing dendrites (arrowheads). The unmineralized bone matrix (asterisk) is on the bottom. E. Detail of the cytoplasm of an active secreting osteoblast. Numerous mitochondria (arrowhead) and developed endoplasmic rough reticulum (arrow) "fulfill" the 
cytoplasm. F. Detail of the cartilage matrix (Ca) and of the bone matrix (Bo) constituted of parallel collagen fibers (arrows). G. Flattened mesenchymal cells (small arrows) forming the periosteum, an active osteoblast (right big arrow) and a Type III preosteocyte (left big arrow) showing dendrites (arrowheads). The unmineralized bone matrix (asterisk) is on the left. H. Fully differentiated osteoblasts form a single layer of cuboidal cells (arrow). Flattened mesenchymal cells (arrowhead) form the periosteum. 
A $13^{2}-8=2+1$

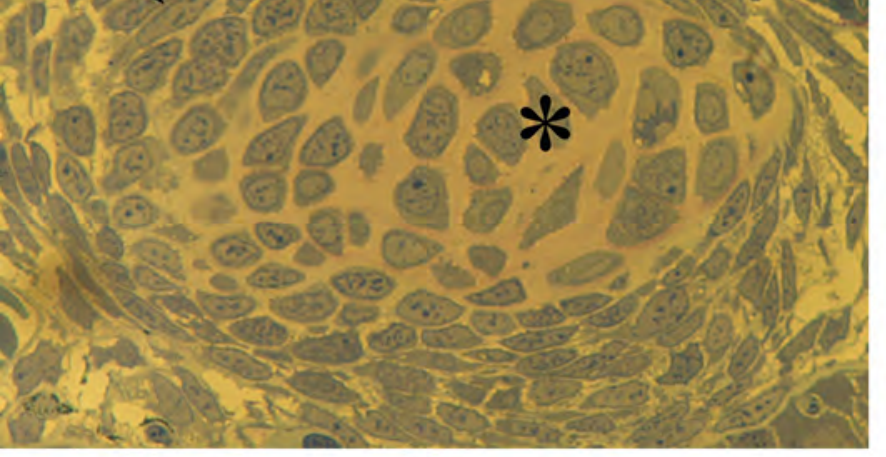

\section{$\mathrm{C} \cdot \mathrm{P}$} (1)

\section{(3) \\ $+2$}

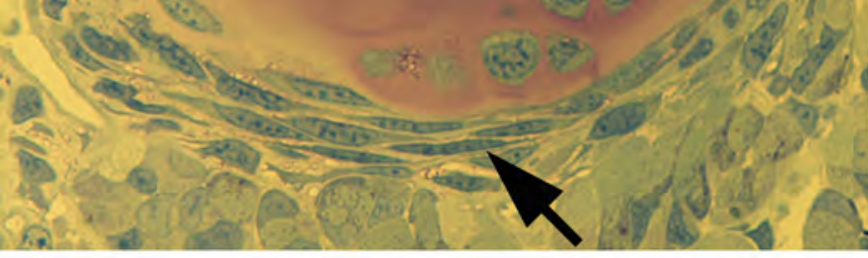

\section{$\mathrm{E}$}
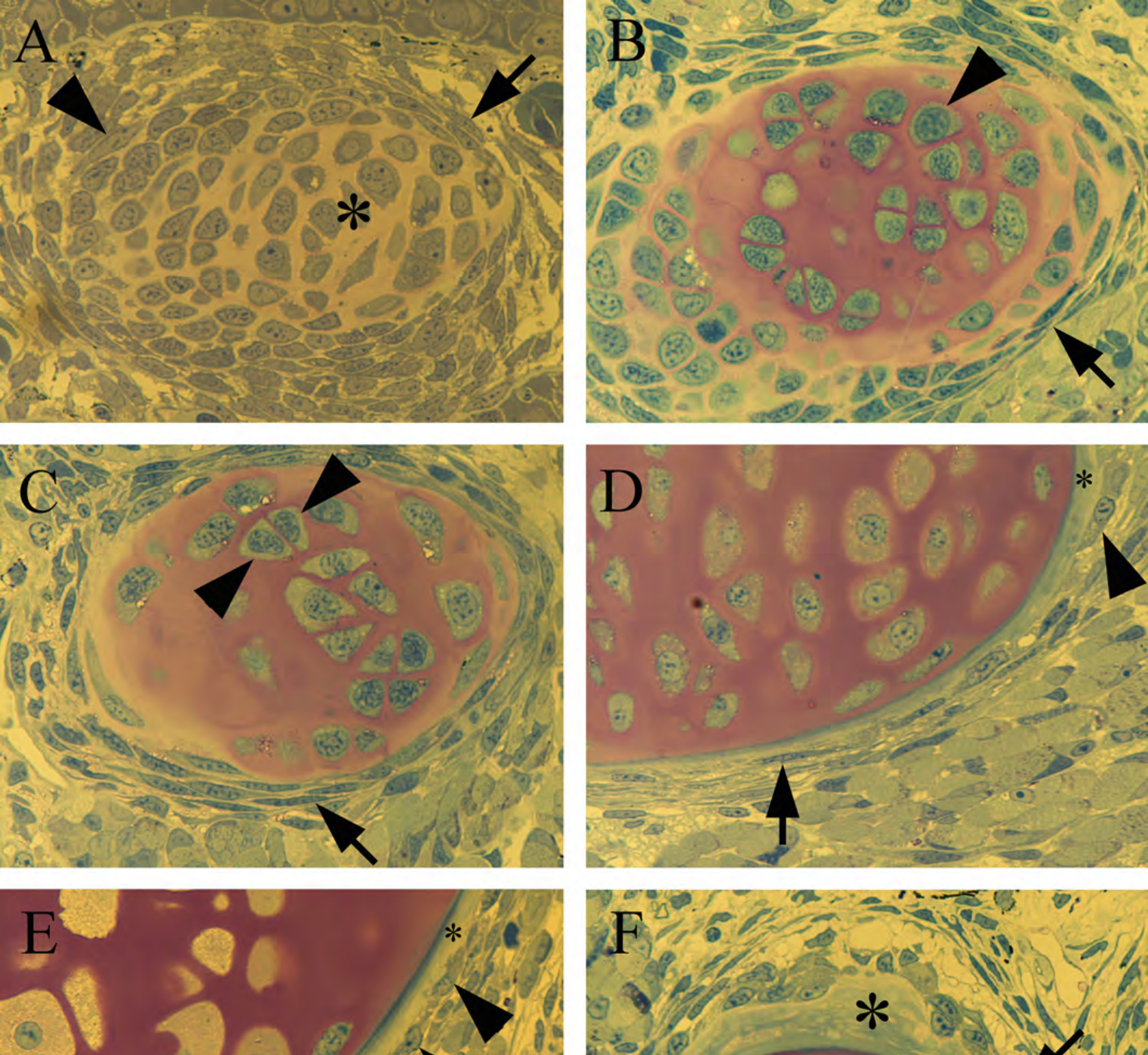

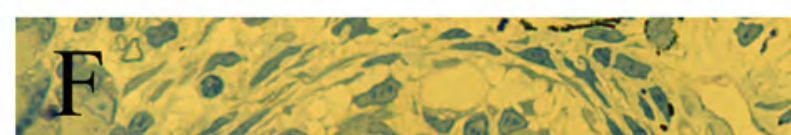

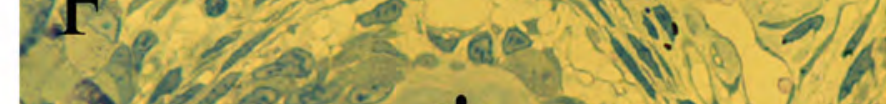

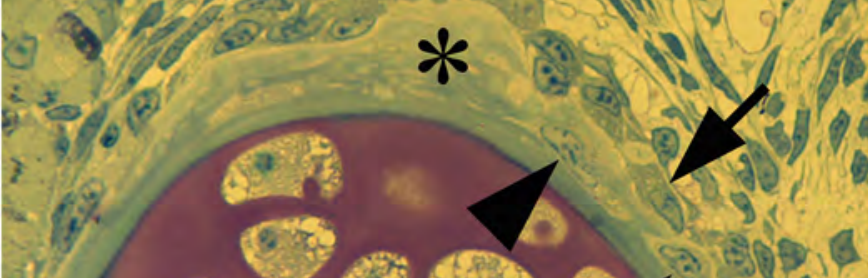

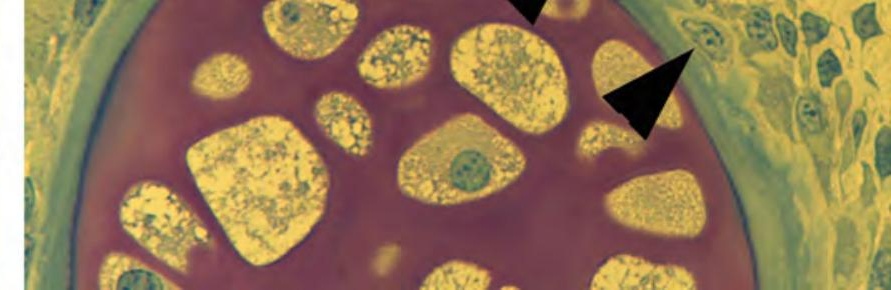

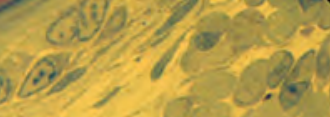
(4) $50 \mu \mathrm{m}$
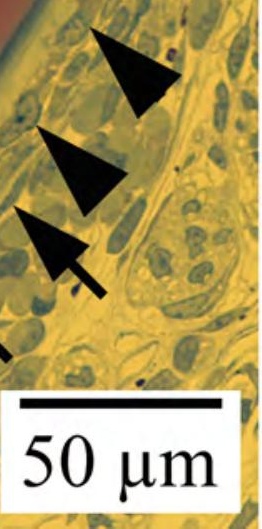


\section{A}
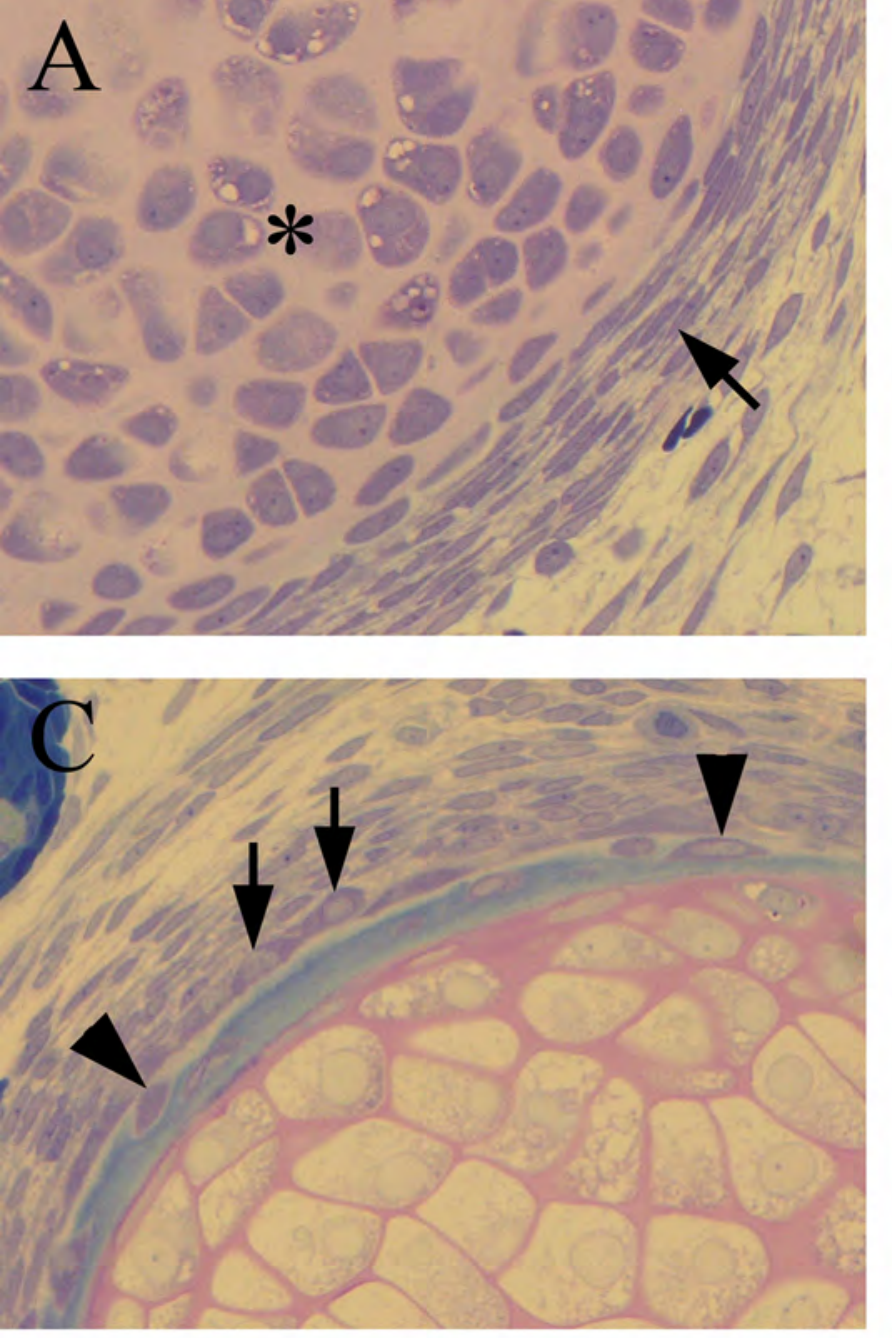

\section{E}
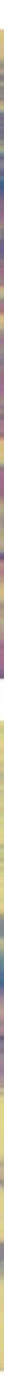

F

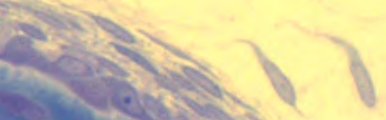

B
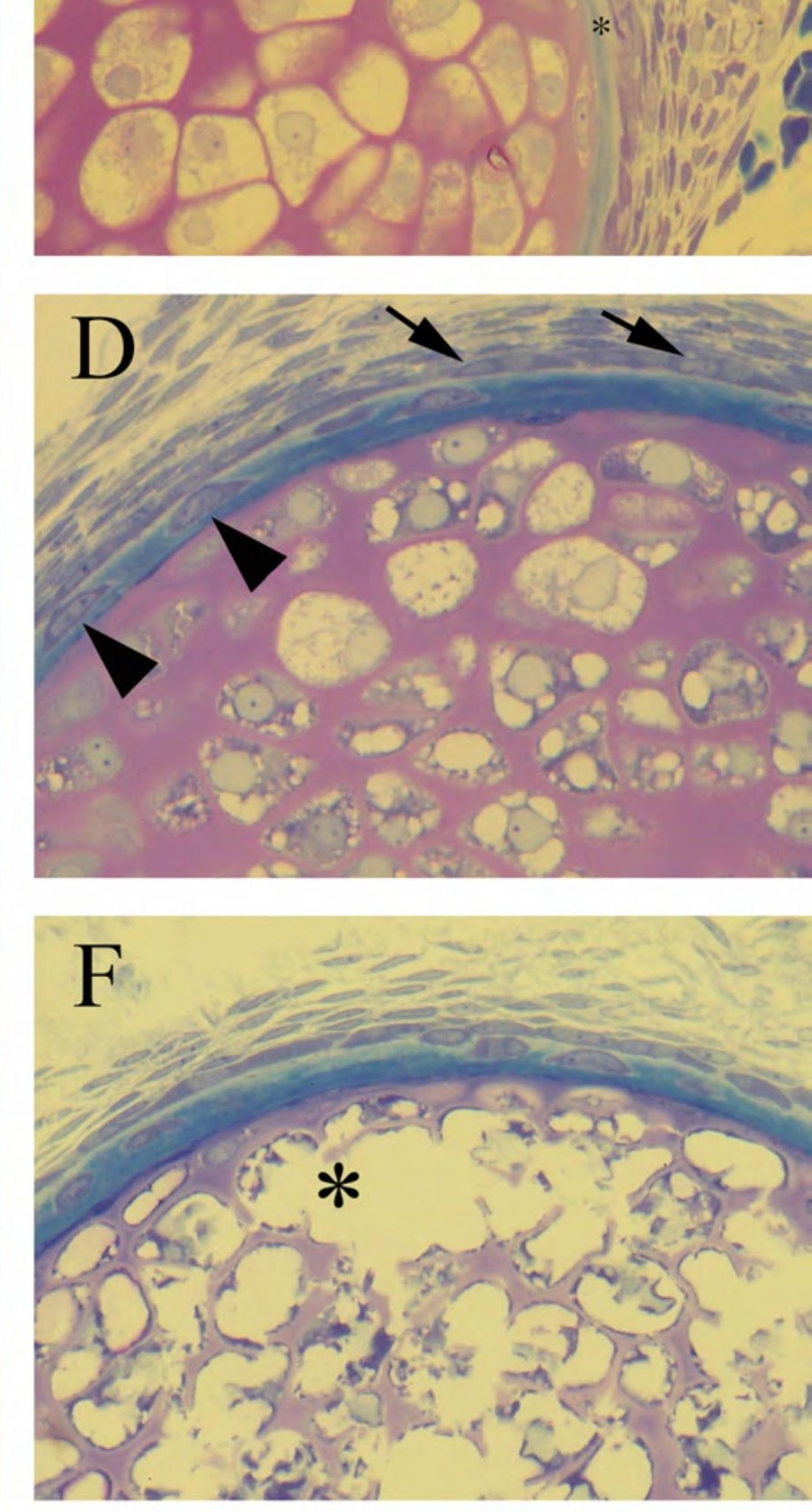

G

$\mathrm{H}$

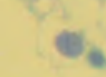
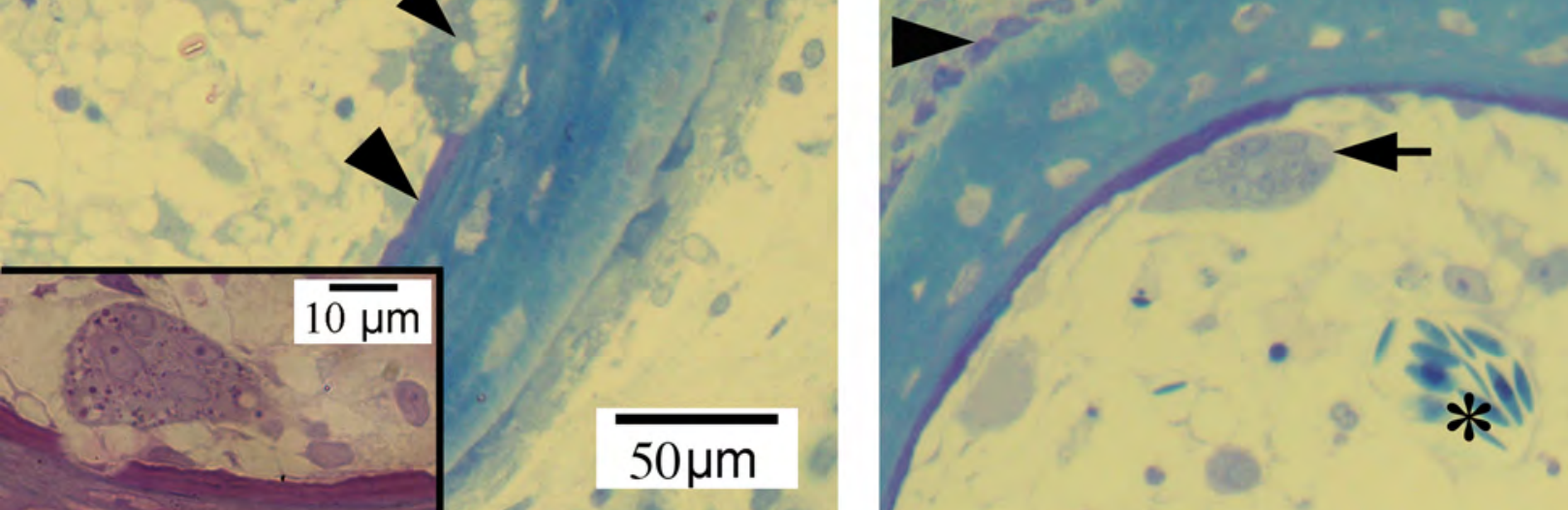


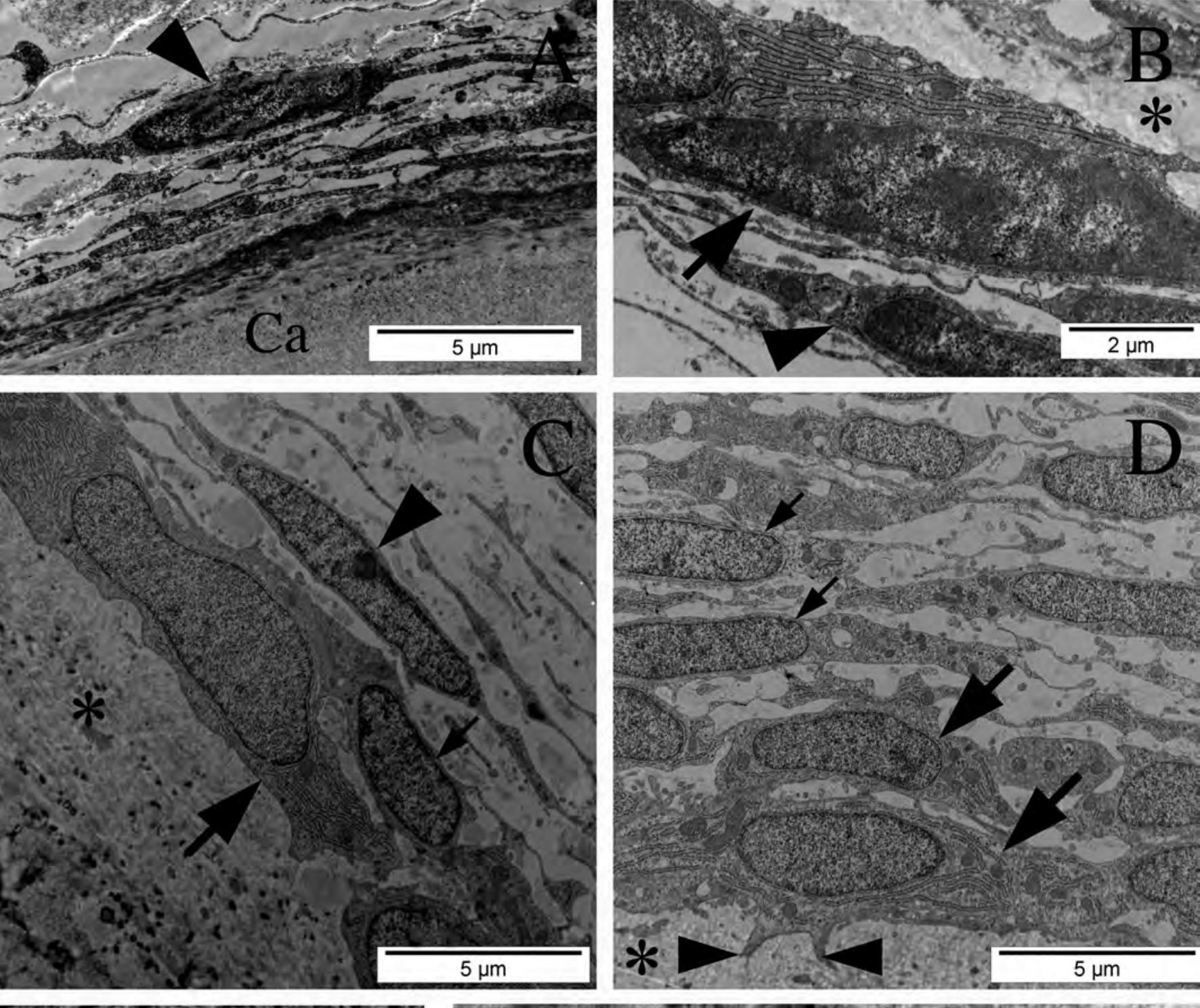

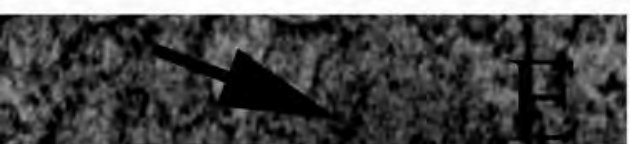
10 (3) $42,0,-3=$ The. (2)

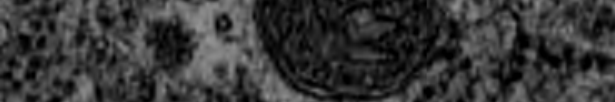
$2 x^{2}+2 x=$

$0,5 \mu \mathrm{m}$ (2) Bo $24 \mathrm{ct}^{\mathrm{Ca}}$ 